\title{
A NEW APPROACH FOR MODELING THE CONTRIBUTION OF NSM FRP STRIPS FOR SHEAR STRENGTHENING OF RC BEAMS
}

Vincenzo Bianco ${ }^{1}$, J.A.O. Barros ${ }^{2}$ and Giorgio Monti ${ }^{3}$

\begin{abstract}
This paper presents the main features of an analytical model recently developed to predict the Near Surface Mounted (NSM) Fiber Reinforced Polymer (FRP) strips shear strength contribution to a Reinforced Concrete (RC) beam throughout the beam's loading process. It assumes that the possible failure modes that can affect the ultimate behavior of an NSM FRP strip comprise: loss of bond (debonding); concrete semi-conical tensile fracture; mixed shallow-semi-cone-plus-debonding and strip tensile fracture. That model was developed by fulfilling equilibrium, kinematic compatibility and constitutive law of both the adhered materials and the bond between them. The debonding process of an NSM FRP strip to concrete was interpreted and closed form equations were derived after proposing a new local bond stress-slip relationship. The model proposed also addressed complex phenomena such as the interaction between the force transferred to the surrounding concrete through bond stresses and concrete fracture as well as the interaction among adjacent strips. The main features of the proposed modeling strategy are shown along with the main underlying physical-mechanical concepts and assumptions. Using recent experimental data, the predictive performance of the model is assessed. The model is also applied to single out the influence of relevant parameters on the NSM technique effectiveness for the shear strengthening of RC beams.
\end{abstract}

CE Database subject headings: FRP; NSM; Shear Strengthening; Concrete Fracture; Debonding; Tensile Rupture.

\section{Introduction}

Strengthening of shear-deficient RC structures by the employment of FRPs, both NSM (De Lorenzis and Nanni 2001, Nanni et al. 2004, Teng et al. 2006, Mohammed Ali et al. 2006) and externally bonded (Triantafillou and Antonopoulos 2000, Teng et al. 2004, Monti and Liotta 2007) is a technique that has been attracting the attention of the academic community in the recent years. Shear strengthening of RC beams by NSM technique consists of

\footnotetext{
${ }^{1}$ PhD Student, Dept. of Structural Engrg. and Geotechnics, Sapienza University of Rome, 00197 Rome, Italy. E-mail: vincenzo.bianco@uniroma1.it.

${ }^{2}$ Associate Professor, Dept. of Civil Engineering, University of Minho, Campus de Azurém, 4810-058 Guimarães, Portugal. E-mail: barros@civil.uminho.pt.

${ }^{3}$ Full Professor, Dept. of Structural Engrg. and Geotechnics, Sapienza University of Rome, 00197 Rome, Italy. E-mail: giorgio.monti@uniroma1.it.
} 
gluing FRP strips by a high performance adhesive into thin shallow slits cut onto the concrete cover of the beam's web lateral faces. Some of the most recent experimental works on NSM (Dias and Barros 2008, Rizzo and De Lorenzis 2009) spotlighted the occurrence of a peculiar failure mode consisting of the progressive detachment and outward expulsion of the concrete cover from the underlying beam core. That failure mode was even more pronounced in case of low strength concrete beams (Dias et al. 2007). It was assumed, taking also advantage of the analogy between adhesive anchors and NSM FRP strips (Bianco et al. 2007a), due to the relative shortage of available test results, that such failure mode can be ascribed to the semi-conical tensile fracture of concrete surrounding each NSM strip (Fig. 1). When principal tensile stresses transferred to the surrounding concrete exceed its tensile strength, concrete fractures along a surface, envelope of the compression isostatics, whose shape can be conveniently assumed as semi-conical. Depending on the relative mechanical and geometrical properties of the materials involved, the possible failure modes affecting the ultimate behavior of NSM strips include: debonding, strip tensile rupture, concrete semi-conical tensile fracture and a mixed shallowsemi-cone-plus-debonding failure mode (Fig. 1). The term debonding is herein adopted to designate loss of bond, which corresponds to a failure either within the adhesive layer or at the FRP strip/adhesive or adhesive/concrete interfaces, as further explained later on. During the loading process of a RC beam under-reinforced in shear, when the principal tensile stresses in a shear critical zone exceed the concrete average tensile strength $f_{c t m}$, some shear cracks originate therein and successively progress towards the web extrados. Those cracks can be thought of as a single Critical Diagonal Crack (CDC) inclined of an angle $\theta$ with respect to the beam longitudinal axis (Fig. 2). The CDC can be schematized as an inclined plane dividing the web into two portions sewn together by the crossing strips (Fig. 2a). At load step $t_{1}$, the two web parts separated by the CDC start moving apart by pivoting around the crack end (point E in Fig. 2b). From that step on, by increasing the applied load, the CDC opening angle $\gamma\left(t_{n}\right)$ progressively widens. The strips crossing the CDC oppose resistance to its widening by anchoring to the surrounding concrete to which they transfer, by bond, the force originating at their intersection with the CDC, $O_{i}^{l}$, and due to the imposed end slip $\delta_{L i}\left[\gamma\left(t_{n}\right)\right]$. The resisting capacity of each strip is provided by its available bond length $L_{f i}$ that is the shorter between the two parts into which the crack divides its actual length $L_{f}$ (Fig. 2b). As the spacing between adjacent strips $s_{f}$ is reduced, their corresponding semi-conical fracture surfaces overlap and the resulting envelope area progressively becomes smaller than the mere summation of each of them (see Fig. 3a). This detrimental interaction between strips can be taken into account by modifying the semi-conical surface pertaining to each strip accordingly. By decreasing the strips 
spacing, the resulting concrete failure surface is almost parallel to the web face of the beam, as confirmed by experimental observations, consisting in the detachment of the concrete cover from the underlying beam core (Dias and Barros 2008, Rizzo and De Lorenzis 2009). Since the position of those semi-conical surfaces is symmetric with respect to the vertical plane passing through the beam axis, the horizontal outward components of the tensile strength vectors distributed throughout their surfaces are balanced only from an overall standpoint but not locally (Fig. 3b). This local unbalance of the horizontal tensile stress components, orthogonal to the beam web face, justifies the outward expulsion of the concrete cover in both the uppermost and lowermost parts of the strengthened sides of the web.

The shear strength contribution provided by a system of NSM FRP strips to a RC beam can be evaluated throughout the loading process by fulfilling equilibrium, kinematic compatibility and constitutive laws of both the intervening materials and the bond between them (Bianco 2008, Bianco et al. 2009). The kinematic mechanism herein adopted envisages, due to the rotation around the CDC end of the two parts into which the web divides, a linear distribution of imposed end slips along the CDC (Fig. 2b). The shear strengthening contribution at the generic load step $V_{f}\left[\gamma\left(t_{n}\right)\right]$ can be evaluated by summing the resistance opposed by each $i$-th strip, as function of the resulting imposed end slip, to the CDC further widening. In correspondence of a generic load step, since concrete around the generic $i$-th strip is not necessarily capable of carrying the stresses transferred to it, it can fracture, up to reaching equilibrium. Contextually to the occurrence of such fractures, the resisting bond length of the strip $L_{R f i}$, that is the amount of its initial available bond length $L_{f i}$ still bonded to concrete, reduces accordingly. Contemporarily, due to the formation of successive co-axial semi-conical concrete fracture surfaces, the total height of the concrete fracture $L_{f i}^{c}$ around the generic $i$-th strip increases accordingly. Thus, the shear strength contribution, at the generic load step, can be evaluated by summing the contribution provided by each strip $V_{f i}\left[\gamma\left(t_{n}\right)\right]$ where this latter can be evaluated by means of an iterative procedure that, given the current value of the imposed end slip and the current value of resisting bond length, searches for the corresponding equilibrium configuration as further explained below.

\section{NSM Shear Strength Contribution}

The algorithm adopted (Fig. 4) takes as input both geometrical and mechanical parameters, i.e.: depth $h_{w}$ and width $b_{w}$ of the strengthened beam web; inclination angle of both the CDC and the strips with respect to the beam longitudinal axis, $\theta$ and $\beta$, respectively; strips spacing measured along the beam axis $s_{f}$; angle $\alpha$ 
between axis and generatrices of the semi-conical fracture surface; concrete average compressive strength $f_{c m}$; strips tensile strength $f_{f u}$ and strips Young's modulus $E_{f}$; thickness $a_{f}$ and width $b_{f}$ of the strip's cross section; values of bond stress $\tau_{0}, \tau_{1}, \tau_{2}$ and slip $\delta_{1}, \delta_{2}, \delta_{3}$ defining the adopted local bond stress-slip relationship; increment $\dot{\gamma}$ and maximum value $\gamma_{\max }$ of the opening angle of the CDC. The algorithm, from the information above and for each $k$-th possible geometrical configuration that the CDC could assume with respect to the strips, determines the NSM strips shear strength contribution as function of the crack opening angle $V_{f, k}\left[\gamma\left(t_{n}\right)\right]$ throughout the loading process ( $t_{n}=t_{1} \rightarrow t_{s}$ where $t_{s}$ is the number of assumed CDC angle increments). In the present work, three geometrical configurations are examined $(k=1,2,3)$, defined as follows: $(k=1)$ the minimum number of strips ( $N_{f, \min }$ ) with the first one located at a distance equal to the spacing from the crack origin; $(k=2)$ an even number of strips $\left(N_{f, e v}\right)$ symmetrically placed with respect to the central point of the segment $\overline{O E}$ (Fig. 2b) which is the trace of the CDC plane on the web face; $(k=3)$ an odd number of strips $\left(N_{f, \text { odd }}\right.$ ) with the central one attaining the maximum $L_{f i}$ by being placed along the axis of the trace of the CDC plane on the web face. For each $k$-th configuration, in correspondence of each $t_{n}$ load step, the contribution provided by each $i$-th strip $V_{f i, k}\left[\gamma\left(t_{n}\right)\right]$ is evaluated and added to the overall shear strength $V_{f, k}\left[\gamma\left(t_{n}\right)\right]$ :

$$
V_{f, k}\left[\gamma\left(t_{n}\right)\right] \leftarrow V_{f, k}\left[\gamma\left(t_{n}\right)\right]+2 \cdot V_{f i, k}\left[\gamma\left(t_{n}\right)\right] \cdot \sin \beta
$$

The contribution provided by the $i$-th strip $V_{f i, k}\left[\gamma\left(t_{n}\right)\right]$ to the overall shear strength is evaluated by taking into consideration: (a) the current value of the imposed end slip $\delta_{L i}\left[\gamma\left(t_{n}\right)\right]$ as results from the imposed kinematic mechanism (Fig. 2b), (b) the current value of the resisting bond length $L_{R f i, k}$, (c) the state of the strip that is whether it has already reached its ultimate state $\left(u_{i, k}=1\right)$ or not $\left(u_{i, k}=0\right)$ and (d) the height of the semi-conical concrete fracture $L_{f j, k}^{c}\left(t_{n}\right)$ around each of the other strips $\left(j=1: N_{f, k}\right.$ and $\left.j \neq i\right)$.

\section{Single strip contribution}

To determine the $i$-th strip contribution $V_{f i}\left[\gamma\left(t_{n}\right)\right]$, an iterative procedure $\left(q_{m}: q_{1} \rightarrow q_{e}\right)$ is carried out (Fig. 5a-b) in order to determine the equilibrium condition $\left(q_{e}\right)$ in the concrete surrounding the $i$-th strip depending, in the most general case, on: a) the imposed end $\operatorname{slip}\left(\delta_{L i}\left(t_{n}\right)\right)$, b) its current state $\left(u_{i}\left(t_{n}\right), L_{R f i}\left(t_{n}\right)\right)$ and c) the current state of concrete fracture regarding all of the other strips ( $L_{f j}^{c}\left(t_{n}\right)$ with $j=1: N_{f, k}$ and $\left.j \neq i\right)$. Nonetheless, that 
iterative procedure can be easily visualized (Fig. 5a-b) referring to the simple case in which: a) the strips are orthogonal to the $\operatorname{CDC}\left(\right.$ e.g. $\left.\theta=\beta=45^{\circ}\right)$ and $\left.\mathrm{b}\right)$ there is no interaction among adjacent strips $\left(e . g . s_{f} \rightarrow \infty\right)$ since, in that case, the progressive concrete fracture capacity $V_{f i}^{c f}\left(X_{i}^{l}\right)$ has an explicit dependence on the value $X_{i}^{l}$ of the abscissa along the strip available bond length. In fact, in that simple case, $V_{f i}^{c f}\left(X_{i}^{l}\right)$ is a parabola (Fig. 5a-b), as further explained below. That iterative procedure is performed as long as neither the surrounding concrete has reached equilibrium nor the strip has ruptured $\left(u_{i}=0\right)$. Within each iteration $\left(q_{m}\right)$, the current bond transfer length $\left(L_{t r, f i}\left[\delta_{L i}\left(t_{n}\right) ; L_{R f i}\left(t_{n} ; q_{m}\right)\right]\right)$ is evaluated as function of the imposed end slip $\delta_{L i}\left(t_{n}\right)$ and the current value of the resisting bond length $L_{R f i}\left(t_{n} ; q_{m}\right)$ and scanned to check if the force progressively transferred through bond $\left(V_{f i}^{b d}\left[\delta_{L i}\left(t_{n}\right) ; L_{R f i}\left(t_{n} ; q_{m}\right) ; x_{i}^{t r}\right]\right)$ exceeds the concrete fracture capacity $\left(V_{f i}^{c f}\left(X_{i}^{l}\right)\right)$. The transfer length is evaluated and discretized in $n_{d}\left(n_{n}: n_{1} \rightarrow n_{d}\right)$ segments and the progressive force transferred by bond is evaluated in their extremities $V_{f i}^{b d}\left[\delta_{L i}\left(t_{n}\right) ; L_{R f i}\left(t_{n} ; q_{m}\right) ; x_{i}^{t r}\left(n_{n}\right)\right]$. The progressive value of $V_{f i}^{c f}$, evaluated in correspondence of the incremental value of the $i$-th strip available bond length $\left(X_{i}^{l}=L_{f i}^{c}+x_{i}^{t r}\left(n_{n}\right)\right)$, is evaluated by spreading the concrete mean tensile strength $f_{c t m}$ over the semi-conical surface with height $X_{i}^{l}$, orthogonally to it in each point and by integrating according to the following formula (Fig. 5c):

$$
V_{f i}^{c f}=\int_{C_{f i}\left(X_{i}^{l} ; \alpha\right)}\left(f_{c t m} \cdot \sin \alpha\right) \cdot d C_{f i}
$$

where $C_{f i}$ concisely denotes the semi-conical surface. Eq. (2) can be reduced to the evaluation of the area of the semi-ellipse $\left(E_{f i}\right)$ intersection of the semi-cone with the crack plane as follows (Bianco et al. 2006, Bianco 2008):

$$
V_{f i}^{c f}=\sin (\theta+\beta) \cdot f_{c t m} \cdot \int_{E_{f i}\left(X_{i}^{l} ; \alpha\right)} d E_{f i}
$$

In general, due to the interaction among adjacent strips, $V_{f i}^{c f}$ also depends on the length of the semi-cones that have already formed along all of the strips as further specified below but, for the simple case in which the strip does not interact with other strips and it is orthogonal to the crack plane (Fig. 5c), Eq. (3) simplifies into: $V_{f i}^{c f}=0.5 \cdot \pi \cdot f_{c t m} \cdot \operatorname{tg}^{2} \alpha \cdot\left(X_{i}^{l}\right)^{2}$. After having scanned the transfer length and having taken note of both the eventual 
occurrence of concrete fracture and the height of the occurred semi-conical fracture, one of the following alternatives might be the case:

- Concrete has fractured but the fracture has not reached the free extremity $\left(L_{f i}^{c}<L_{f i}\right)$. In this case, it is taken note of the up-dated height of the cone $\left(L_{f i}^{c}\left(t_{n} ; q_{m+1}\right)\right)$, of the resisting bond length $\left(L_{R f i}\left(t_{n} ; q_{m+1}\right)\right)$ and iteration is performed $\left(q_{m} \leftarrow q_{m+1}\right)$;

- Concrete has fractured and the fracture has reached the free extremity $\left(L_{f i}^{c}=L_{f i}\right)$. Note is taken of the updated value of both semi-cone height and resisting bond length as above and of the $i$-th strip having reached its ultimate state $\left(u_{i} \leftarrow 1\right)$. The $i$-th strip shear strength contribution is $V_{f i}\left(t_{n}\right)=V_{f i}^{c f}\left(L_{f i}\right)$;

- Concrete has not fractured and the actual value of bond-transferred force is lower than the strip tensile rupture capacity $\left(V_{f i}^{b d}\left(n_{d}\right)<V_{f}^{t r}\right)$. In this case equilibrium in concrete is reached and there is no need to iterate and the strip strength is equal to the actual value of the force transferred by bond $\left(V_{f i}\left(t_{n}\right)=V_{f i}^{b d}\left(n_{d}\right)\right)$;

- Concrete has not fractured and the actual value of bond-transferred force is higher or equal to the strip tensile rupture capacity $\left(V_{f i}^{b d}\left(n_{d}\right) \geq V_{f}^{t r}\right)$. In this case equilibrium in concrete is reached and there is no need to iterate, the strip strength is equal to the strip tensile fracture capacity $\left(V_{f i}\left(t_{n}\right)=V_{f}^{t r}\right)$ and note can be taken that the strip has reached its ultimate state $\left(u_{i, k} \leftarrow 1\right)$.

\section{Progressive bond transferred force}

The progressive value of the force transferred by the $i$-th strip through bond stresses to the surrounding concrete along the current value of the resisting bond length $V_{f i}^{b d}\left[\delta_{L i}\left(t_{n}\right) ; L_{R f i}\left(t_{n} ; q_{m}\right) ; x_{i}^{t r}\left(n_{n}\right)\right]$ and due to the imposed end slip $\delta_{L i}\left(t_{n}\right)$ is obtained by fulfilling equilibrium, constitutive law of the adhered materials and the local bond stress-slip relationship between them. The local bond stress-slip relationship herein proposed to simulate the subsequent phases undergone by bond during the loading process is composed of four different linear branches (Fig. 6). Those phases, representing the physical phenomena occurring in sequence within the adhesive layer by increasing the imposed end slip, are labeled as: elastic, softening, softening friction and free slipping. The first rigid branch $\left(0-\tau_{0}\right)$ represents the overall initial shear strength of the joint independent of the deformability of the adhesive layer and attributable to the micro-mechanical and chemical properties of the involved materials and interfaces. In fact, the parameter $\tau_{0}$ is the average of the following physical entities encountered in sequence by 
forces flowing from the strip to the surrounding concrete, i.e.: adhesion at the strip-adhesive interface, cohesion within the adhesive itself, and adhesion at the adhesive-concrete interface. From $\tau_{0}$ up to the peak strength $\tau_{1}$, a macro-mechanical strength due to the adhesive layer elastic stiffness adds to the constant adhesive-cohesive strength. That macro-mechanical strength due to the elastic stiffness of the intact adhesive layer can be conveniently modeled by a linear elastic behavior. Approaching the peak strength, the adhesive fractures along diagonal planes orthogonal to the tension isostatics as outlined by Sena-Cruz and Barros (2004) by means of post-test optical microscope photos and finite element materially nonlinear analysis (Sena-Cruz 2004). During the subsequent softening phase, force is transferred from the strip to the surrounding concrete by the resulting diagonal micro-struts. Anyway, throughout the softening phase, by increasing the imposed slip, the adhesive at the extremities of those struts progressively deteriorates, so that, by increasing the imposed slip, micro-cracks parallel to the strip start to appear at both the strip-adhesive and adhesive-concrete interfaces. Approaching the softening friction phase, the softening resisting mechanism is gradually replaced by friction and micro-mechanical interlock along those micro-cracks. Nonetheless, even those mechanisms undergo softening due to progressive material degradation. When the resisting force provided by friction is exhausted, those micro-cracks result in smooth discontinuities. The free slipping phase follows, during which the strip keeps being pulled out without having to overcome any opposing restraint left. For computational ease, both softening and softening-frictional behaviors are modeled as linear. The resulting analytical relationship is the following:

$$
\tau(\delta)=\left\{\begin{array}{lr}
\tau_{0}+\frac{\tau_{1}-\tau_{0}}{\delta_{1}} \cdot \delta & 0 \leq \delta \leq \delta_{1} \\
\tau_{1}-\frac{\tau_{1}-\tau_{2}}{\delta_{2}-\delta_{1}} \cdot\left(\delta-\delta_{1}\right) & \delta_{1}<\delta \leq \delta_{2} \\
\tau_{2}-\frac{\tau_{2}}{\delta_{3}-\delta_{2}} \cdot\left(\delta-\delta_{2}\right) & \delta_{2}<\delta \leq \delta_{3} \\
0 & \delta>\delta_{3}
\end{array}\right.
$$

Note that, even if at a first sight the consideration of $\tau_{0}$ may seem an useless and sterile redundancy, from a strict analytical standpoint it is indeed absolutely necessary to obtain an explicit equation providing the value of the bond transfer length as function of the imposed and slip and the resisting bond length (Bianco et al. 2007b, 2009).

Among the parameters defining the turning points of the local bond stress slip relationship, the adhesive-cohesive term $\tau_{0}$ is the one that more markedly depends on the micro-mechanical and chemical properties of the composite, of the adhesive and of the concrete surfaces as well as on the adhesive layer thickness. For better characterizing the influence of those aspects, a closer scale investigation is deemed as necessary. Moreover, all 
of the parameters defining the local bond stress-slip relationship should not be considered as having universally valid values but, on the contrary, should be determined on the basis of the mechanical-chemical and geometrical parameters characterizing the specific case at hand but, in that respect, further research is required.

The current value of the bond transfer length $L_{t r, f i}\left(L_{R f i} ; \delta_{L i}\right)$ is discretized in $n_{d}$ segments and the corresponding values of the progressive force transferred to the surrounding concrete through bond $V_{f i}^{b d}\left[L_{R f i} ; \delta_{L i} ; x_{i}^{t r}\left(n_{n}\right)\right]$ are evaluated.

It was recently assumed (Bianco et. al 2008), also by means of comparison between experimental recordings and analytical predictions, that the employment of bond and the successive and progressive loss of bond (debonding), under an increasing end slip to an NSM FRP strip, can be thought of as a "constant wave", i.e., an invariant distribution of tangential stress progressing from the loaded end inwards, towards the free extremity. From an analytical standpoint, for the given value of $\delta_{L i}$, the governing differential equation is first solved for an infinite value of the resisting bond length, thus determining the trends of slip $\delta(x)$, tangential stress $\tau(x)$ and axial stress in the strip $\sigma_{f}(x)$ along the corresponding transfer length $L_{t r}\left(\delta_{L i}\right)$. After that, the actual value of the transfer length $L_{t r, f i}\left(L_{R f i} ; \delta_{L i}\right)$ is determined, as the minimum between the necessary transfer length $L_{t r}\left(\delta_{L i}\right)$ and $L_{R f i}$ and then discretization and integration are performed as further explained below. The governing differential equation is the following (Bianco et al. 2009):

$$
\frac{d^{2} \delta}{d x^{2}}-\tau[\delta(x)] \cdot J_{1}=0 \quad \text { with } J_{1}=\frac{L_{p}}{A_{f}} \cdot\left(\frac{1}{E_{f}}+\frac{A_{f}}{A_{c} \cdot E_{c}}\right)
$$

where $L_{p}=2 \cdot b_{f}+a_{f}$ is the effective bond perimeter of the strip cross-section, $A_{f}=a_{f} \cdot b_{f}$ is the strip crosssection area and $A_{c}=s_{f} \cdot b_{w} \cdot 0.5$ is the area of the cross-section of the concrete prism that is the amount of the surrounding concrete attributed to the $i$-th strip. Eq. (5) is obtained by taking into consideration: a) the equilibrium relationships: $d \sigma_{f}(x) / d x-\tau(x) \cdot L_{p} / A_{f}=0$ and $\sigma_{f}(x) \cdot A_{f}+\sigma_{c}(x) \cdot A_{c}=0 ;$ b) the constitutive equations for the adhesive layer $\tau=\tau(\delta)$ and for the two adhering materials: $\sigma_{f}=E_{f} \cdot d u_{f} / d x$ and $\sigma_{c}=E_{c} \cdot d u_{c} / d x ;$ c) kinematic compatibility $\delta(x)=u_{f}(x)-u_{c}(x)$, where $u_{f}(x)$ and $u_{c}(x)$ are the local displacements of strip and concrete, respectively.

Once the relationship $\delta(x)$ has been obtained by solving Eq. (5) with the convenient boundary conditions, the equations for the axial stress in the strip and the tangential stress along this latter can be deduced as follows: 
$\sigma_{f}(x)=J_{2} \cdot \frac{d \delta}{d x} \quad$ with $J_{2}=\frac{E_{f} \cdot E_{c} \cdot A_{c}}{E_{c} \cdot A_{c}+E_{f} \cdot A_{f}}$

and:

$\tau(x)=J_{3} \cdot \frac{d^{2} \delta}{d x^{2}} \quad$ with $J_{3}=\frac{E_{f} \cdot A_{f} \cdot E_{c} \cdot A_{c}}{L_{p} \cdot\left(E_{c} \cdot A_{c}+E_{f} \cdot A_{f}\right)}$

\section{Solution of Bond for an infinite resisting bond length}

\section{Elastic phase}

When the imposed end slip is $\delta_{L i} \leq \delta_{1}$, the equation of the interfacial slip, obtained by solving Eq. (5) written in the local reference system $o x^{e}$ originating in the leftward unloaded extremity of the transfer length (Fig. 7a) and imposing the boundary conditions $\delta^{e}=0$ at $x^{e}=0$ and $\delta^{e}=\delta_{L i}$ at $x^{e}=L_{t r}^{e}$, is the following:

$\delta^{e}\left(x^{e}\right)=C_{1}^{e} \cdot e^{\lambda \cdot x^{e}}+C_{2}^{e} \cdot e^{-\lambda \cdot x^{e}}-\frac{\tau_{0} \cdot J_{1}}{\lambda^{2}}$

where $C_{1}^{e}\left(L_{t r}^{e}\right), C_{2}^{e}\left(L_{t r}^{e}\right)$ and $\lambda$ are integration constants whose analytical expressions are herein omitted, for the sake of brevity, but they can be found elsewhere (Bianco et. al 2007b, 2008). By imposing the equilibrium condition $L_{p} \cdot \int_{0}^{L_{r r}^{e}} \tau^{e}\left(x^{e}\right) \cdot d x^{e}=A_{f} \cdot \sigma_{f}\left(L_{t r}^{e}\right)$, the equation of the transfer length for the first phase can be obtained as function of the imposed slip:

$L_{t r}\left(\delta_{L i}\right)=L_{t r}^{e}\left(\delta_{L i}\right)=\frac{1}{\lambda} \cdot \operatorname{arcosh} \frac{B^{e}}{2 \cdot A^{e}}$

with $A^{e}$ and $B^{e}$ constants (Bianco et. al 2007b, 2008). The transfer length at the end of the elastic phase $L_{t r 1}$ and the corresponding value of force transferred to concrete $V_{1}^{b d}$, both invariants for given input parameters, are obtained by imposing $\delta_{L i}=\delta_{1}$ :

$L_{t r 1}=L_{t r}^{e}\left(\delta_{1}\right) ; V_{1}^{b d}=V^{b d, e}\left(\delta_{1}\right)=L_{p} \cdot \int_{0}^{L_{r r 1}} \tau^{e}\left(x^{e}\right) \cdot d x^{e}$ 
When the imposed end slip is $\delta_{1}<\delta_{L i} \leq \delta_{2}$, the equation of the interfacial slip, obtained by solving Eq. (5) written in the reference system $o x^{s}$ originating at the point of the bond length where slip is equal to $\delta_{1}$ (Fig. 7b), with boundary conditions $\delta^{s}=\delta_{1}$ at $x^{s}=0$ and $\delta^{s}=\delta_{L i}$ at $x^{s}=L_{t r}^{s}$, is the following:

$\delta^{s}\left(x^{s}\right)=C_{1}^{s} \cdot \sin \left(\beta_{1} \cdot x^{s}\right)+C_{2}^{s} \cdot \cos \left(\beta_{1} \cdot x^{s}\right)+\frac{\tau_{1} \cdot J_{1}}{\beta_{1}^{2}}+\delta_{1}$

with $C_{1}^{s}\left(L_{t r}^{s}\right), C_{2}^{s}$ and $\beta_{1}$ integration constants (Bianco et. al 2007b, 2008). The equation of the transfer length $L_{t r}^{s}\left(\delta_{L i}\right)$ corresponding to the amount of the infinite bond length undergoing softening is:

$L_{t r}^{s}\left(\delta_{L i}\right)=\frac{1}{\beta_{1}} \cdot \phi+\frac{1}{\beta_{1}} \cdot \arcsin \frac{C^{s}}{\left[\left(A^{s}\right)^{2}+\left(B^{s}\right)^{2}\right]^{1 / 2}}$

with $A^{s}, B^{s}, C^{s}$ and $\phi$ constants (Bianco et. al 2007b, 2008). The overall transfer length, for $\delta_{1}<\delta_{L i} \leq \delta_{2}$, is then:

$L_{t r}\left(\delta_{L i}\right)=L_{t r 1}+L_{t r}^{s}\left(\delta_{L i}\right)$

The maximum value of the transfer length that can undergo softening and the relevant value of the force transferred to the surrounding concrete are the following invariants:

$$
L_{t r 2}=L_{t r}^{s}\left(\delta_{2}\right) ; V_{2}^{b d}=V^{b d, s}\left(\delta_{2}\right)=L_{p} \cdot \int_{0}^{L_{t r 2}} \tau^{s}\left(x^{s}\right) \cdot d x^{s}
$$

\section{Softening Friction Phase}

When the imposed slip is larger than the value at which softening friction begins, $\delta_{2}<\delta_{L i} \leq \delta_{3}$, the equation for the interfacial slip, obtained by solving Eq. (5) written for a reference system $o x^{s f}$ originating at the point of the infinite bond length where slip is equal to $\delta_{2}$ (Fig. 7c) and with boundary conditions $\delta^{s f}=\delta_{2}$ at $x^{s f}=0$ and $\delta^{s f}=\delta_{L i}$ at $x^{s f}=L_{t r}^{s f}$, is:

$\delta^{s f}\left(x^{s f}\right)=C_{1}^{s f} \cdot \sin \left(\gamma_{1} \cdot x^{s f}\right)+C_{2}^{s f} \cdot \cos \left(\gamma_{1} \cdot x^{s f}\right)+\delta_{3}$

with $C_{1}^{s f}\left(L_{t r}^{s f}\right), C_{2}^{s f}$ and $\gamma_{1}$ constants (Bianco et. al 2007b, 2008). The expression of the transfer length $L_{t r}^{s f}\left(\delta_{L i}\right)$ corresponding to the amount of length undergoing softening friction is:

$$
L_{t r}^{s f}\left(\delta_{L i}\right)=\frac{1}{\gamma_{1}} \cdot \psi+\frac{1}{\gamma_{1}} \cdot \arcsin \frac{C^{s f} \cdot}{\left[\left(A^{s f}\right)^{2}+\left(B^{s f}\right)^{2}\right]^{1 / 2}}
$$


with $A^{s f}, B^{s f}, C^{s f}$ and $\psi$ constants (Bianco et. al 2007b, 2008). The overall transfer length, for $\delta_{2}<\delta_{L i} \leq \delta_{3}$, is:

$$
L_{t r}\left(\delta_{L i}\right)=L_{t r 1}+L_{t r 2}+L_{t r}^{s f}\left(\delta_{L i}\right)
$$

The maximum value of the infinite bond length that can undergo softening friction and the relevant value of the force transferred to the surrounding concrete are:

$$
L_{t r 3}=L_{t r}^{s f}\left(\delta_{3}\right) ; V_{3}^{b d}=V^{b d, s f}\left(\delta_{3}\right)=L_{p} \cdot \int_{0}^{L_{t r 3}} \tau^{s f}\left(x^{s f}\right) \cdot d x^{s f}
$$

\section{Free Slipping Phase}

When the imposed slip is larger than the value at which free slipping begins, $\delta_{L i}>\delta_{3}$, the equation for the interfacial slip, obtained by solving Eq. (5) written for a reference system $o x^{f_{s}}$ originating at the point of the bond length where slip is equal to $\delta_{3}$ (Fig. 8) and with boundary conditions $\delta^{f s}=\delta_{3}$ at $x^{f s}=0$ and $\delta^{f s}=\delta_{L i}$ at $x^{f_{s}}=L_{t r}^{f_{s}}$, is:

$\delta^{f_{s}}\left(x^{f_{s}}\right)=C_{1}^{f_{s}} \cdot x^{f_{s}}+C_{2}^{f_{s}}$

with $C_{1}^{f s}\left(L_{t r}^{f s}\right)$ and $C_{2}^{f s}$ constants (Bianco et. al 2007b, 2008). The expression of the transfer length $L_{t r}^{f s}\left(\delta_{L i}\right)$ corresponding to the amount of length undergoing free slipping is:

$$
L_{t r}^{f s}\left(\delta_{L i}\right)=J_{3} \cdot L_{p} \cdot \frac{\delta_{L i}-\delta_{3}}{V_{1}^{b d}+V_{2}^{b d}+V_{3}^{b d}}
$$

The overall transfer length, for $\delta_{L i}>\delta_{3}$, and the force transferred by bond to the surrounding concrete, are:

$$
L_{t r}\left(\delta_{L i}\right)=\sum_{p=1}^{3} L_{t r p}+L_{t r}^{f s}\left(\delta_{L i}\right) ; V^{b d}\left(\delta_{L i}\right)=\sum_{p=1}^{3} V_{p}^{b d}
$$

\section{Solution of Bond for the actual value of the resisting bond length}

After having solved the governing differential equation for the current value of the imposed slip $\delta_{L i}$ and having thereby determined the corresponding transfer length $L_{t r}\left(\delta_{L i}\right)$ and trend of bond stress $\tau(x)$ for the case of an infinite resisting bond length (Fig. 9a), the actual value of the transfer length $L_{t r, f i}\left(L_{R f i} ; \delta_{L i}\right)$ is determined as the minimum between $L_{R f i}\left(t_{n} ; q_{m}\right)$ and $L_{t r}\left(\delta_{L i}\right)$. The transfer length is then discretized and the progressive value of 
force transferred by bond is determined by integrating the tangential stress according to the following general formulation:

$V_{f i}^{b d}\left[x_{i}^{t r}\left(n_{n}\right)\right]=L_{p} \cdot\left[\int \tau^{s f}\left(x^{s f}\right) \cdot d x^{s f}+\int \tau^{s}\left(x^{s}\right) \cdot d x^{s}+\int \tau^{e}\left(x^{e}\right) \cdot d x^{e}\right]$

where the integration extremities are omitted since they depend on both the phase undergone by the free end and the current value of the progressive abscissa $x_{i}^{t r}\left(n_{n}\right)$ along the transfer length (Fig. 9b1-2).

\section{Concrete Fracture Capacity}

In the most general case in which the $i$-th strip progressive concrete fracture capacity $V_{f i}^{c f}\left[X_{i}^{l}\left(n_{n}\right)\right]$ is influenced by the semi-conical fracture surfaces that have already occurred around all of the strips up to that moment ( $L_{f j}^{c}\left(t_{n} ; q_{m}\right) \forall j=1, ., N_{f k}$ and $j \neq i$ ) (Fig. $5 \mathrm{~d}$ ) its evaluation becomes more complex. However, the simplification adopted in Eq. (3) that reduces the evaluation of the semi-conical surface area to the area of the semi-ellipse (intersection of the semi-conical surface with the CDC plane) is extremely powerful to correctly quantify interaction among strips. In the most general case (Fig. 5d and Fig. 10) such interaction can be either mono-directional - longitudinal or transversal - or bi-directional. Longitudinal interaction can occur when, due to the reduced spacing with respect to the height of the beam's web, the semi-cones associated to adjacent strips located on the same side of the web, and consequently their corresponding semi-ellipses, overlap along their major semi-axis (see for instance the semi-ellipses 1 and 3 of the example of Fig. 10). Transversal interaction can occur when, for slender beam cross-sections of high $h_{w} / b_{w}$ ratio, the semi-ellipses symmetrically placed on the opposite sides of the web, intersect each other along their minor semi-axis (see the semi-ellipse 2 of Fig. 10). In this latter case, the area of the $i$-th semi-ellipse is limited, upwards, by the line $Y=b_{w} / 2$, i.e. the trace, on the CDC plane (with reference system $O X Y$ ), of the vertical plane passing through the beam axis. In case bidirectional interaction occurred, the area on the CDC plane associated to the $i$-th strip would be composed of two terms: $\mathcal{A}_{f i}^{\text {nlin }}$, limited upwards by the non-linear branch of the corresponding semi-ellipse $Y_{i}(X)$ and another, $\mathcal{A}_{f i}^{\text {lin }}$, limited by the line $Y=b_{w} / 2$. Hence, due to the bi-directional interaction, the area of the semiellipse associated to the $i$-th strip is calculated as follows:

$\int_{E_{f i}\left(X_{i}^{l} ; \alpha_{f i}\right)} d E_{f i}=\left(\mathcal{A}_{f i}^{\text {nlin }}+\mathcal{A}_{f i}^{l i n}\right)$ 
The two areas $\mathcal{A}_{f i}^{\text {lin }}$ and $\mathcal{A}_{f i}^{\text {nlin }}$ are evaluated by a geometrical closed-form algorithm (Bianco et al. 2006, Bianco 2008) that briefly consists of: (1) writing the equation of each $j$-th semi-ellipse associated to the $j$-th strip's latest semi-conical fracture surface $\left(j: 1 \rightarrow N_{f, k}\right)$, if it has formed, in the CDC reference system; (2) evaluating and storing in some auxiliary vectors the abscissa of the points that might constitute integration extremities for the $i$-th semi-ellipse; (3) suitably selecting the integration extremities for both the linear and nonlinear integration range of the $i$-th semi-ellipse and (4) integrating (Fig. 10). For the sake of brevity, all of the analytical details are herein omitted but they can be found elsewhere (Bianco et al. 2006, Bianco 2008).

\section{Model Appraisal}

The proposed model was applied to the RC beams tested by Dias and Barros (2008), by Dias et al. (2007) and by Dias (2008). The beams tested in the first two experimental programs (series I and II) were T cross-section RC beams characterized by the same test set-up with the same ratio between the shear span and the beam effective depth $(a / d=2.5)$, the same amount of longitudinal reinforcement, the same kind of CFRP strips and epoxy adhesive and they differed for the concrete mechanical properties. In fact, the first experimental program was characterized by a concrete mean compressive strength $f_{c m}$ of $31.1 \mathrm{MPa}$, while the second by $18.6 \mathrm{MPa}$. Both series presented different configurations of NSM strips, in terms of both inclination $\beta$ and spacing $s_{f}$. The first program also included beams characterized by a different amount of existing steel stirrups (see Table 1). The beams tested in the third experimental program (series III) were characterized by the same test set up, but with a different shear aspect ratio $(a / d=3.3)$ and distinct concrete mechanical properties $\left(f_{c m}=59.4 M P a\right)$. Some of them were also subject to pre-cracking (their label includes a letter F). The details of the beams taken to appraise the predictive performance of the developed model are listed in Table 1. Those beams are characterized by the following common geometrical and mechanical parameters: $b_{w}=180 \mathrm{~mm} ; h_{w}=300 \mathrm{~mm} ; f_{f u}=2952 \mathrm{MPa}$ (for the series I and II) and $f_{f u}=2847.9 \mathrm{MPa}$ (for the series III); $E_{f}=166 \mathrm{GPa}$ (for the series I and II) and $E_{f}=174.3 \mathrm{GPa}$ (for the series III); $a_{f}=1.4 \mathrm{~mm} ; b_{f}=10.0 \mathrm{~mm}$. The parameters characterizing the adopted local bond stress-slip relationship, being the average values of those obtained in a previous investigation by curve fitting of experimental recordings (Bianco et al. 2007b and 2009), are: $\tau_{0}=2.0 \mathrm{MPa} ; \tau_{1}=20.1 \mathrm{MPa}$; $\tau_{2}=9.0 \mathrm{MPa} ; \delta_{1}=0.07 \mathrm{~mm} ; \delta_{2}=0.83 \mathrm{~mm} ; \delta_{3}=14.1 \mathrm{~mm}$. The CDC inclination angle $\theta$ adopted in the simulations, listed in Table 1 for all the analyzed beams, is the one experimentally observed by inspecting the 
crack patterns. The angle $\alpha$ was assumed equal to $28.5^{\circ}$, being the average of values obtained in a previous investigation (Bianco et al. 2006) by back analysis of experimental data. The two parameters characterizing the loading process are: $\dot{\gamma}=0.01^{\circ}$ and $\gamma_{\max }=1.0^{\circ}$. Concrete average tensile strength $f_{\text {ctm }}$ was calculated from the average compressive strength by means of the formulae of the CEB Fib Model Code 1990 resulting in 2.45 $M P a$, 1.45 MPa and 4.17 MPa for the series I, II and III, respectively.

Comparison between the numerical results and experimental recordings, for some of the beams listed in Table 1, are plotted in Fig. 11. From that comparison, a satisfactory data-fitting performance of the proposed model, in terms of prediction of the NSM shear strength contribution $V_{f, k}$, arises, regardless of the different concrete mechanical properties, inclination of the strips, their spacing along the beam axis, amount of existing stirrups and shear span to depth ratio.

As to the influence of the amount of existing steel stirrups, it is expected that, when their amount is increased, the semi-conical concrete fracture surfaces can not penetrate deep inside the web core but remain shallower. The proposed model may be easily adjusted to take into consideration that aspect even if the interaction with existing stirrups is deemed a marginal aspect. In fact in practice, most of beams that need a retrofitting intervention are characterized by a small amount of existing stirrups. However, the amount of existing stirrups also affects the occurred CDC inclination angle i.e.: in general, the larger the amount of reinforcement, the less inclined the CDC. Thus, the model ends up giving reasonable estimates of the experimental recordings even for the beams with larger amount of existing stirrups, when the experimentally observed CDC inclination $\theta^{\exp }$ is adopted (Table 1). Further research is, in this respect, needed.

When the spacing between adjacent strips is increased, the difference between the peak shear strength contribution obtained in the three different geometrical configurations increases (see Fig. 11), as expected.

The typical graph of shear strength contribution as function of the CDC opening angle $V_{f}\left[\gamma\left(t_{n}\right)\right]$ is characterized by abrupt decays which correspond to the failure of the strips. The peculiar behavior of a RC beam strengthened in shear by NSM technique can be easily explained referring to one of those beams, as for instance 2S-7LI45-II beam, whose cracking scenario, both numerically predicted and experimentally recorded, is reported in Fig. 12. The first strips to fail are those of shorter available bond lengths that generally fail in the first stages of the loading process, like for instance: the $1^{\text {st }}\left(\gamma=0.02^{\circ}\right)$ and the $5^{\text {th }}\left(\gamma=0.03^{\circ}\right)$ of the $1^{\text {st }}$ configuration (Fig. 12a); the $1^{\text {st }}$ and $6^{\text {th }}\left(\gamma=0.01^{\circ}\right)$ and the $2^{\text {nd }}\left(\gamma=0.02^{\circ}\right)$ of the $2^{\text {nd }}$ configuration (Fig. 12b). Those failures are not so evident in the corresponding graph (Fig. 11) since, in the first load steps, the contribution provided by 
the strips with a longer available bond length is increasing and relatively much higher. When a strip fails at a higher stage of the loading process, the corresponding decay in the load carrying capacity, is much more evident, like it happens, for instance: for the $2^{\text {nd }}$ strip of the $1^{\text {st }}$ configuration at $\gamma=0.07^{\circ}$, the $3^{\text {rd }}$ of the $2^{\text {nd }}$ configuration at $\gamma=0.07^{\circ}$ or the $3^{\text {rd }}$ strip of the $3^{\text {rd }}$ configuration at $\gamma=0.19^{\circ}$. The former two are mixed shallow-semi-coneplus-debonding failures and the third is characterized by a semi-conical concrete fracture that reaches the inner tip. After those failures $\left(p_{11}, p_{21}, p_{32}\right)$, the corresponding graphs, show a different trend (Fig. 11): in the first two cases (configurations $k=1$ and $k=2)$ a relative maximum follows $\left(p_{12} \cong p_{22}\right)$, while in the third case (configuration $k=3$ ) the shear carrying capacity decreases continuously. The behavior of the first two cases is due to the fact that, when the last fracture occurs, the remaining strips still have a resisting bond length larger than the required transfer length and their contribution can still increase before the occurrence of complete debonding. The behavior of the third case is due to the fact that, when the $3^{\text {rd }}$ central strip fails, the $2^{\text {nd }}$ and the $4^{\text {th }}$, had already failed by mixed failure, therefore the overall carrying capacity keeps diminishing up to the complete debonding of their left resisting bond lengths.

The numerical modeling strategy herein proposed also lets parametric studies be carried out in order to assess the influence of all the involved parameters on the NSM shear strength contribution. Herein, for the sake of brevity, only a small parametric study is presented (Fig. 13a-b) that aims at singling out, even by means of comparison between numerical predictions and experimental recordings, the influence of the strips spacing for beams with strips at $60^{\circ}$ and with two different kinds of concrete. It arises that, as expected, the higher the concrete mechanical properties, the higher the shear carrying capacity, for the same value of spacing between adjacent strips. It can also be gathered that, by reducing the spacing between adjacent strips, due to the increase of the number of strips effectively crossing the $\mathrm{CDC}$, the shear strength contribution increases even if, as highlighted in Fig. $13 \mathrm{c}$ for the $3^{\text {rd }}$ configuration only (with $\beta=60^{\circ} ; f_{c m}=31.1 \mathrm{MPa} ; \theta=45^{\circ}$ and all the other parameters with the same values adopted for the series I, II), the smaller the spacing, the higher is the group effect. This latter can be defined as the decrease of shear strength contribution with respect to an ideal situation in which (Fig. 14), the same system of strips, characterized by the real value of the spacing $s_{f}$, the same available bond lengths and the same imposed end slips, are spaced out, along the CDC, at such an extent that they do not interact any longer between each other. The corresponding increase in shear strength contribution increases up to a maximum ideal value beyond which any further increase of the ideal spacing between adjacent strips does not produce any further increase in carrying capacity. This can be also gathered from Fig. 13d in which the ideal 
trend is plotted as function of the ideal spacing for the real configuration of strips at $s_{f}=75 \mathrm{~mm}$. The detrimental group effect increases by reducing the spacing between strips (Fig. 13c).

\section{Conclusions}

The need to provide a rational explanation to the observed peculiar failure mode affecting the behavior, at ultimate, of RC beam strengthened in shear by the NSM technique led to the development of a comprehensive numerical model to simulate the NSM shear strength contribution throughout the loading process. The comparison between the numerical predictions and the experimental recordings showed a satisfactory level of accuracy of the proposed model, especially if one considers that: the model neglects the softening behavior of concrete in tension, the high scatter affecting concrete tensile strength, which indeed, was indirectly evaluated from compression tests results.

The application of that model also allowed to identify and thoroughly describe some complex phenomena, such as the group effect between adjacent strips. Despite its relative complexity, the proposed model can be usefully applied to single out relevant information for designers interested in applying such front-line technique.

\section{Acknowledgements}

The authors of the present work wish to acknowledge the support provided by the "Empreiteiros Casais", S\&P $®$, degussa ${ }^{\circledR}$ Portugal, and Secil (Unibetão, Braga). The study reported in this paper forms a part of the research program "SmartReinforcement - Carbon fibre laminates for the strengthening and monitoring of reinforced concrete structures" supported by ADI-IDEIA, Project n 13-05-04-FDR-00031. Also, this work was carried out under the auspices of the Italian DPC-ReLuis Project (repertory n. 540), Research Line 8, whose financial support is greatly appreciated. 


\section{Notation}

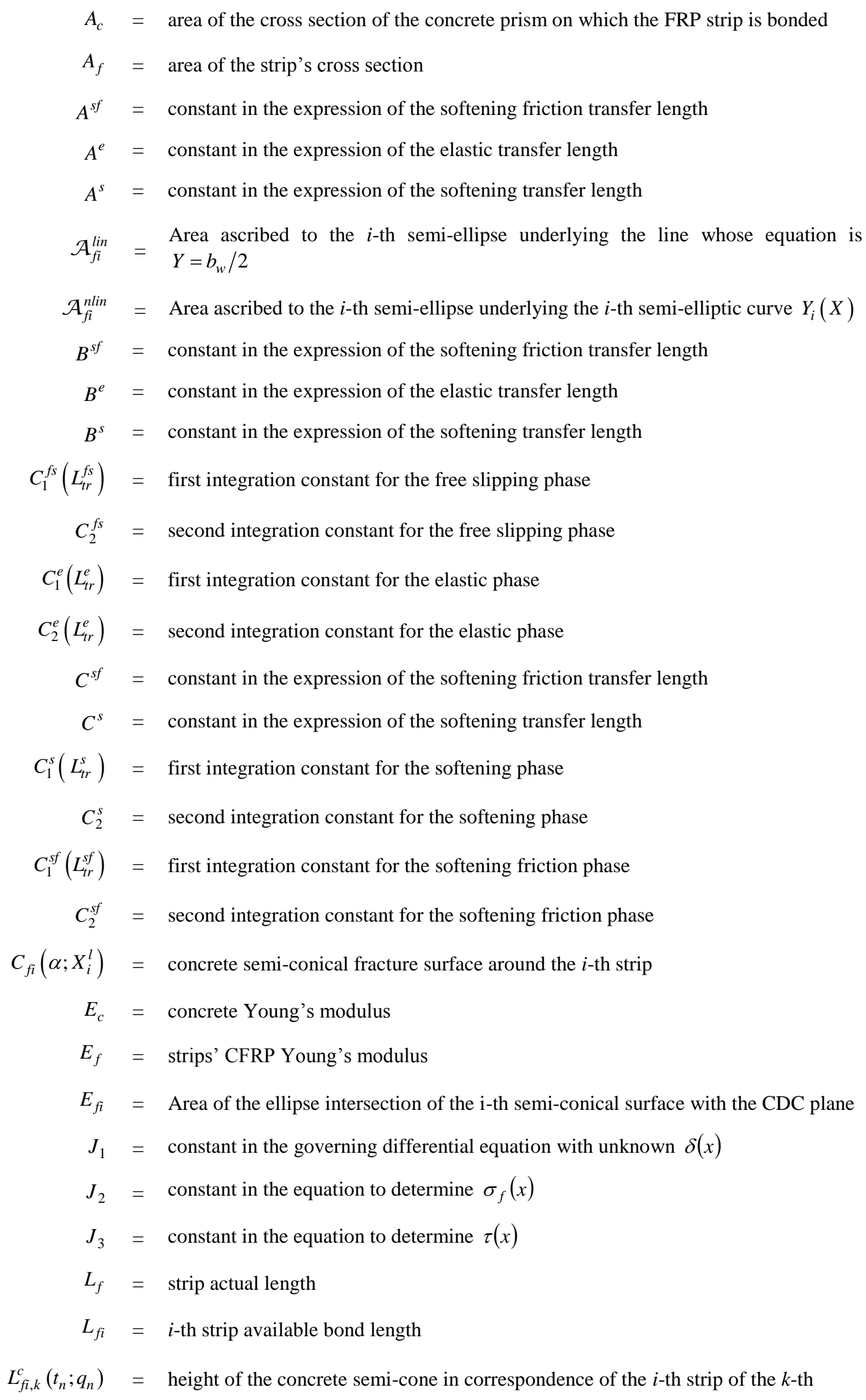




$$
\begin{aligned}
& \text { geometrical configuration } \\
& L_{p} \quad=\text { effective perimeter of the strip cross section } \\
& L_{R f i, k}\left(t_{n} ; q_{m}\right) \quad=\quad \text { resisting bond length of the } i \text {-th strip of the } k \text {-th geometrical configuration } \\
& L_{t r, f i}\left(L_{R f i} ; \delta_{L i}\right)=\begin{array}{l}
\text { transfer length of the } i \text {-th strip, function of the corresponding imposed end slip and } \\
\text { resisting bond length }
\end{array} \\
& L_{t r 1}=\text { maximum invariant value of transfer length that can undergo elastic phase } \\
& L_{t r 2}=\text { maximum invariant value of transfer length that can undergo softening } \\
& L_{t r 3}=\text { maximum invariant value of transfer length that can undergo softening friction } \\
& L_{t r}\left(\delta_{L i}\right)=\text { value of the necessary transfer length for an infinite value of the resisting bond } \\
& \text { length and function of the imposed end slip only } \\
& L_{t r}^{f_{s}}\left(\delta_{L i}\right)=\begin{array}{l}
\text { amount of a transfer length for an infinite resisting bond length undergoing free } \\
\text { slipping }
\end{array} \\
& L_{t r}^{e}\left(\delta_{L i}\right)=\text { amount of a transfer length for an infinite bond length undergoing elastic phase } \\
& L_{t r}^{s f}\left(\delta_{L i}\right)=\text { softening frictional amount of a transfer length for an infinite resisting bond length } \\
& L_{t r}^{s}\left(\delta_{L i}\right)=\text { amount of a transfer length for an infinite bond length undergoing softening } \\
& N_{f, e v}=\text { even (integer) number of strips that can effectively cross the CDC } \\
& N_{f, k}=\begin{array}{l}
\text { Number of strips effectively crossing the CDC in the k-th geometrical } \\
\text { configuration }
\end{array} \\
& N_{f, \min }=\text { minimum (integer) number of strips that can effectively cross the CDC } \\
& N_{f, o d d}=\text { odd (integer) number of strips that can effectively cross the CDC } \\
& \text { OXYZ = crack plane reference system } \\
& O_{i}^{l} X_{i}^{l}=\text { reference axis along the } i \text {-th strip available bond length } L_{f i} \\
& V_{c}=\text { vertex of the } i \text {-th concrete semi-conical fracture surface } \\
& V_{f i}^{b d}\left(\delta_{L i} ; L_{R f} ; x_{i}^{t r}\right)=\text { progressive force transferred through bond stresses along the current value of the } \\
& \text { resisting bond length as function of the current value of the imposed end slip } \\
& V_{f i}^{c f}\left(X_{i}^{l}\right) \quad \text { progressive concrete tensile fracture capacity along the } i \text {-th strip available bond } \\
& \text { length } L_{f i} \\
& V_{f i}^{b d}\left(L_{R f i} ; \delta_{L i} ; x_{i}^{t r}\right)=\text { progressive value of the force transferred to concrete by the } i \text {-th strip } \\
& V_{f i}^{b d}\left(L_{R f i} ; \delta_{L i}\right)=\text { actual value of force transferred to concrete through bond by the } i \text {-th strip } \\
& V_{f i, k}\left[\gamma\left(t_{n}\right)\right]=\text { Resistance opposed by the } \mathrm{i} \text {-th strip of the k-th geometrical configuration to the } \\
& \text { CDC opening angle } \gamma\left(t_{n}\right) \\
& V_{f, k}\left[\gamma\left(t_{n}\right)\right]=\text { NSM shear strength contribution as function of the imposed end slip for the } k \text {-th } \\
& \text { geometrical configuration } \\
& V^{b d, e}\left(\delta_{L i}\right)=\text { force transferred by bond in the elastic phase for an infinite bond length }
\end{aligned}
$$




$$
\begin{aligned}
& V^{b d, s}\left(\delta_{L i}\right)=\text { force transferred by bond in softening phase for an infinite bond length } \\
& V^{b d, s f}\left(\delta_{L i}\right)=\text { force transferred by bond in the softening friction phase for an infinite bond length } \\
& V_{1}^{d b}=\text { value of force transferred by bond along the elastic transfer length } L_{t r 1} \\
& V_{2}^{d b}=\text { value of force transferred by bond along the softening transfer length } L_{t r 2} \\
& V_{3}^{d b}=\text { value of force transferred by bond along the softening friction transfer length } L_{t r} \\
& X_{f i}=\text { position of the i-th strip along the CDC } \\
& X_{i}^{l}=\text { Reference axis along the i-th strip available bond length } \\
& Y_{i}(X)=\begin{array}{l}
\text { general expression of the equation of the } i \text {-th semi-ellipse in the } O X Y \text { crack plane } \\
\text { reference system }
\end{array} \\
& a=\text { beam shear span } \\
& a_{f}=\text { strip cross section's thickness } \\
& b_{f}=\text { strip cross section's width } \\
& b_{w}=\text { beam web width } \\
& d=\text { beam cross section effective depth } \\
& f_{c m}=\text { concrete average compressive strength } \\
& f_{c t m}=\text { concrete average tensile strength } \\
& f_{f u}=\text { FRP tensile strength } \\
& h_{w}=\text { beam web depth } \\
& i=\text { counter of the strips effectively crossing the CDC } \\
& j=\text { counter of the strips effectively crossing the CDC } \\
& k={ }_{\mathrm{CDC}} \\
& n_{d}=\text { number of segments into which the bond transfer length is discretized } \\
& n_{d}=\text { counter of the number of segments into which the bond transfer length is } \\
& \text { discretized } \\
& n_{1}=\text { first segment of the discretization of the bond transfer length } \\
& o^{f_{s}} x^{f_{s}}=\text { reference axis along the amount of the infinite strip in free slipping phase } \\
& o^{e} x^{e}=\text { reference axis along the amount of the infinite strip in bond elastic phase } \\
& o^{s} x^{s}=\text { reference axis along the amount of the infinite strip in softening phase } \\
& o^{s f} x^{s f}=\text { reference axis along the amount of the infinite strip in softening friction phase } \\
& o_{i}^{t r} x_{i}^{t r}=\text { reference axis along the strip's transfer length } \\
& q_{e}=\text { iteration in correspondence of which equilibrium is attained in the concrete } \\
& q_{m}=\text { m-th iteration } \\
& s_{f}=\text { strips spacing along the beam axis }
\end{aligned}
$$




$$
\begin{aligned}
& t_{1}=\text { load step at which the critical diagonal crack starts widening } \\
& t_{n}=\text { generic } \mathrm{n} \text {-th load step } \\
& t_{s}=\text { number of assumed CDC opening angle increments } \\
& u_{c}(x)=\text { local displacement of the concrete surrounding the strip } \\
& u_{f}(x)=\text { local displacement of the strip } \\
& \text { logical parameter indicating if the } i \text {-th strip of the k-th geometrical configuration } \\
& u_{i, k}=\text { has reached } u_{i, k}=1 \text { or not } u_{i, k}=0 \text { its ultimate state } \\
& \alpha=\text { angle between the axis and the generatrices of the concrete semi-conical farctures } \\
& \beta=\text { FRP strips inclination angle with respect to the beam longitudinal axis } \\
& \beta_{1}=\text { constant entering the governing differential equation for softening phase } \\
& \phi=\text { constant in the expression of the softening transfer length } \\
& \delta(x)=\text { slip along the strip's resisting bond length } \\
& \delta_{1}=\text { slip corresponding to peak of local bond stress-slip relationship } \\
& \delta_{2}=\text { slip corresponding to start of softening-friction in the local bond relationship } \\
& \delta_{3}=\begin{array}{l}
\text { slip corresponding to the start of free-slipping in the local bond stress-slip } \\
\text { relationship }
\end{array} \\
& \delta_{L i}\left[\gamma\left(t_{n}\right)\right]=\text { imposed slip at the loaded extremity of the i-th strip } \\
& \delta^{f_{s}}\left(x^{f_{s}}\right)=\text { slip-abscissa relationship along the amount of transfer length in free slipping phase } \\
& \delta^{e}\left(x^{e}\right)=\text { slip-abscissa relationship along the amount of transfer length in elastic phase } \\
& \delta^{s}\left(x^{s}\right)=\text { slip-abscissa relationship along the amount of transfer length in the softening phase } \\
& \delta^{s f}\left(x^{s f}\right)=\begin{array}{l}
\text { slip-abscissa relationship along the amount of transfer length in the softening } \\
\text { friction }
\end{array} \\
& \gamma_{1}=\text { constant in the expression of the softening frictional transfer length } \\
& \gamma\left(t_{n}\right)=\text { critical diagonal crack opening angle } \\
& \dot{\gamma}=\text { imposed angle increment for the critical diagonal crack opening angle } \gamma \\
& \gamma_{\max }=\text { maximum value of the CDC opening angle } \\
& \lambda=\text { constant entering the governing differential equation for elastic phase } \\
& \theta=\text { shear crack inclination angle } \\
& \sigma_{c}(x)=\text { concrete axial stress along the resisting bond length } \\
& \sigma_{f}(x)=\text { Strip axial stress along the resisting bond length } \\
& \tau(\delta)=\text { local bond stress-slip relationship }
\end{aligned}
$$




$$
\begin{aligned}
\tau^{e}\left(x^{e}\right) & =\begin{array}{l}
\text { bond stress-abscissa relationship along the amount of transfer length in elastic } \\
\text { phase }
\end{array} \\
\tau^{f s}\left(x^{f s}\right) & =\begin{array}{l}
\text { bond stress-abscissa relationship along the amount of transfer length in softening } \\
\text { frictional phase }
\end{array} \\
\tau^{s}\left(x^{s}\right) & =\begin{array}{l}
\text { bond stress-abscissa relationship along the amount of transfer length in softening } \\
\text { phase }
\end{array} \\
\tau^{s f}\left(x^{s f}\right)= & \text { bond stress-abscissa relationship along the amount of transfer length in softening } \\
\tau(x) & =\text { bond stress along the strip resisting bond length } \\
\tau_{0} & =\text { adhesive-cohesive initial bond strength } \\
\tau_{1} & =\text { peak stress of the local bond stress-slip relationship } \\
\tau_{2} & =\text { shear strength at the beginning of softening-friction of local bond } \\
\psi & =\text { constant in the expression of the softening frictional transfer length }
\end{aligned}
$$




\section{References}

Bianco, V., Barros, J.A.O., Monti, G., (2006). "Shear Strengthening of RC beams by means of NSM laminates: experimental evidence and predictive models”, Technical report 06-DEC/E-18, Dep. Civil Eng., School Eng. University of Minho, Guimarães- Portugal.

Bianco, V., Barros, J.A.O., Monti, G., (2007a). "A new approach for modeling the NSM shear strengthening contribution in reinforced concrete beams", FRPRCS-8, University of Patras, Greece,16-18 July, ID 8-12.

Bianco, V., Barros, J.A.O., Monti, G., (2007b). "Shear Strengthening of RC beams by means of NSM strips: a proposal for modeling debonding”, Technical report 07-DEC/E-29, Dep. Civil Eng., School Eng. University of Minho, Guimarães- Portugal.

Bianco, V., Barros, J.A.O., Monti, G., (2009). "Bond Model of NSM FRP strips in the context of the Shear Strengthening of RC beams", ASCE Journal of Structural Engineering, in press.

Bianco, V., (2008). "Shear Strengthening of RC beams by means of NSM FRP strips: experimental evidence and analytical modeling”, PhD Thesis, Dept. of Structural Engrg. and Geotechnics, Sapienza University of Rome, Italy, submitted on December 2008.

CEB-FIP Model Code 90, (1993) Bulletin d'Information N²13/214, Final version printed by Th. Telford, London, (1993; ISBN 0-7277-1696-4; 460 pages).

De Lorenzis, L. and Nanni, A., (2001), "Shear strengthening of Reinforced Concrete Beams with Near-Surface Mounted Fiber-Reinforced Polymer Rods”, ACI Structural Journal, Vol. 98, N.1, pp. 60-68.

Dias, S.J.E. (2008). "Experimental and anlytical research in the shear strengthening of reinforced concreet beams using the near surface mounted technique with CFRP strips", PhD Thesis, Department of Civil Engineering, University of Minho, Guimarães-Portugal, in Portuguese.

Dias, S.J.E., Bianco, V., Barros, J.A.O., Monti, G., (2007). "Low strength concrete T cross section RC beams strengthened in shear by NSM technique”, Workshop-Materiali ed Approcci Innovativi per il Progetto in Zona Sismica e la Mitigazione della Vulnerabilità delle Strutture, University of Salerno, Italy, 12-13 February.

Dias, S.J.E. and Barros, J.A.O., (2008). "Shear Strengthening of T Cross Section Reinforced Concrete Beams by Near Surface Mounted Technique”, Journal of Composites for Construction, ASCE, Vol. 12, No. 3, pp. 300-311.

Mohammed Ali, M.S., Oehlers, D.J., Seracino, R. (2006). "Vertical shear interaction model between external FRP transverse plates and internal stirrups", Engineering Structures 28, 381-389. 
Monti, G., Liotta, Marc'Antonio, (2007) “Tests and design equations for FRP-strengthening in shear", Construction and Building Materials, Vol. 21, No. 4, 799-809.

Nanni, A., Di Ludovico, M., Parretti, R., (2004) "Shear Strengthening of a PC Bridge Girder with NSM CFRP Rectangular Bars”, Advances in Structural Engineering, Vol. 7, No. 4.

Rizzo, A., De Lorenzis, L., (2009). "Behaviour and capacity of RC beams strengthened in shear with NSM FRP reinforcement”, Construction and Building Materials 23 1555-1567.

Sena-Cruz, J.M. (2004). "Strengthening of concrete structures with near-surface mounted CFRP laminate strips" PhD Thesis, Department of Civil Engineering, University of Minho, Guimarães-Portugal.

Sena-Cruz, J.M., Barros, J.A.O., (2004). "Bond between near-surface mounted CFRP laminate strips and concrete in structural strengthening”, Journal of Composites for Construction, ASCE, Vol. 8, No. 6, pp. 519-527.

Teng J.G., Lam L., Chen J.F. (2004). "Shear strengthening of RC beams with FRP composites”, Prog Struct Eng Mater 2004, 6:173-84.

Teng, J.G., De Lorenzis, L., Wang, Bo, Rong, Li, Wong, T.N. and Lik, Lam, (2006) “Debonding failures of RC beams Strengthened with Near Surface Mounted CFRP Strips”, Journal of Composites for Constructions, ASCE, March/April, pp.92-105.

Triantafillou, T.C., and Antonopoulos, C.P., (2000), "Design of concrete flexural members strengthened in shear with FRP”, ASCE Journal of Composites for Construction, 4(4), pp. 198-205. 


\section{TABLE CAPTIONS}

Table 1. Values of the parameters characterizing beams adopted to appraise the proposed model.

Table 1. Values of the parameters characterizing beams adopted to appraise the proposed model.

\begin{tabular}{|c|c|c|c|c|c|c|c|c|}
\hline $\begin{array}{l}\text { Beam } \\
\text { Label }\end{array}$ & $\theta^{\exp }$ & $\begin{array}{c}\beta \\
\circ\end{array}$ & $\begin{array}{l}s_{f} \\
m m\end{array}$ & $\begin{array}{c}\text { Steel } \\
\text { Stirrups }\end{array}$ & $\begin{array}{c}V_{f, 1}^{\max } \\
k N\end{array}$ & $\begin{array}{c}V_{f, 2}^{\max } \\
k N\end{array}$ & $\begin{array}{c}V_{f, 3}^{\max } \\
k N\end{array}$ & $\begin{array}{c}V_{f}^{\exp } \\
k N\end{array}$ \\
\hline 2 S-3LV-I & 40 & 90 & 267 & $\$ 6 / 300 \mathrm{~mm}$ & 18.53 & 6.46 & 55.33 & 22.20 \\
\hline 2S-5LV-I & 40 & 90 & 160 & “ & 52.33 & 26.42 & 55.34 & 25.20 \\
\hline 2S-8LV-I & 36 & 90 & 100 & “ & 68.58 & 58.88 & 64.33 & 48.60 \\
\hline 2S-3LI45-I & 45 & 45 & 367 & “ & 35.10 & 15.41 & 45.73 & 29.40 \\
\hline 2S-5LI45-I & 45 & 45 & 220 & “ & 46.11 & 49.14 & 45.74 & 41.40 \\
\hline 2S-8LI45-I & 36 & 45 & 138 & “ & 75.89 & 79.71 & 78.73 & $40.20 *$ \\
\hline 2S-3LI60-I & 33 & 60 & 325 & “ & 50.69 & 18.90 & 51.68 & 35.40 \\
\hline 2S-5LI60-I & 36 & 60 & 195 & “ & 36.37 & 36.59 & 48.55 & 46.20 \\
\hline 2S-7LI60-I & 33 & 60 & 139 & “ & 52.98 & 63.07 & 67.58 & 54.60 \\
\hline 2S-7LV-II & 46 & 90 & 114 & $\phi 6 / 300 \mathrm{~mm}$ & 26.72 & 31.84 & 35.59 & 28.32 \\
\hline 2S-4LI45-II & 40 & 45 & 275 & “ & 25.06 & 21.89 & 37.30 & 33.90 \\
\hline 2S-7LI45-II & 30 & 45 & 157 & “ & 49.36 & 47.13 & 45.95 & 48.00 \\
\hline 2S-4LI60-II & 40 & 60 & 243 & “ & 21.31 & 15.04 & 29.38 & 33.06 \\
\hline 2S-6LI60-II & 27 & 60 & 162 & “ & 42.79 & 37.54 & 39.45 & 42.72 \\
\hline 4S-7LV-II & 46 & 90 & 114 & $\phi 6 / 180 \mathrm{~mm}$ & 26.72 & 31.84 & 35.59 & $6.90 *$ \\
\hline 4S-4LI45-II & 40 & 45 & 275 & “ & 25.06 & 21.89 & 37.30 & 26.04 \\
\hline 4S-7LI45-II & 40 & 45 & 157 & “ & 40.58 & 37.48 & 40.63 & 31.56 \\
\hline 4S-4LI60-II & 40 & 60 & 243 & “ & 21.31 & 15.04 & 29.38 & 25.08 \\
\hline 4S-6LI60-II & 30 & 60 & 162 & “ & 38.92 & 35.46 & 36.71 & 35.10 \\
\hline 3S-5LI45-III & 30 & 45 & 275 & $\phi 6 / 300 \mathrm{~mm}$ & 59.74 & 59.55 & 70.01 & 66.10 \\
\hline 3S-5LI45F1-III*** & 23 & 45 & 275 & “ & 83.05 & 86.96 & 81.15 & 85.75 \\
\hline 3S-5LI45F2-III** & 30 & 45 & 275 & “ & 59.74 & 59.55 & 70.01 & 65.35 \\
\hline 5S-5LI45-III & 28 & 45 & 275 & $\phi 6 / 200 \mathrm{~mm}$ & 78.24 & 59.55 & 72.01 & 74.90 \\
\hline 5S-5LI45F-III** & 28 & 45 & 275 & “ & 78.24 & 59.55 & 72.01 & 74.90 \\
\hline 3S-9LI45-III & 32 & 45 & 157 & $\phi 6 / 300 \mathrm{~mm}$ & 109.88 & 109.32 & 98.30 & 101.85 \\
\hline 5S-9LI45-III & 32 & 45 & 157 & $\phi 6 / 200 \mathrm{~mm}$ & 109.88 & 109.32 & 98.30 & 108.90 \\
\hline 3S-5LI60-III & 26 & 60 & 243 & $\$ 6 / 300 \mathrm{~mm}$ & 71.74 & 76.20 & 62.81 & 69.00 \\
\hline 5S-5LI60-III & 25 & 60 & 243 & $\phi 6 / 200 \mathrm{~mm}$ & 68.48 & 77.44 & 63.79 & 73.35 \\
\hline 5S-5LI60F-III** & 25 & 60 & 243 & “ & 68.48 & 77.44 & 63.79 & 72.55 \\
\hline 3S-8LI60-III & 22 & 60 & 162 & $\phi 6 / 300 \mathrm{~mm}$ & 112.82 & 119.58 & 112.25 & 112.30 \\
\hline 5S-8LI60-III & 19 & 60 & 162 & $\phi 6 / 200 \mathrm{~mm}$ & 123.34 & 122.74 & 132.00 & 122.45 \\
\hline 3S-6LV-III & 45 & 90 & 180 & $\phi 6 / 300 \mathrm{~mm}$ & 58.24 & 26.62 & 66.53 & 39.58 \\
\hline 3S-10LV-III & 32 & 90 & 114 & “ & 97.50 & 82.41 & 85.21 & 83.25 \\
\hline
\end{tabular}

I) beams tested by Dias \& Barros (2006) and characterized by a/d equal to 2.5 and $f_{c m}$ equal to $31.1 \mathrm{MPa}$;

II) beams tested by Dias et al. (2007) and characterized by a/d equal to 2.5 and $f_{c m}$ equal to $18.6 \mathrm{MPa}$; III) beams tested by Dias (2008) and characterized by a/d equal to 3.3 and $f_{c m}$ equal to $59.4 \mathrm{MPa}$.

* beams whose experimental value of NSM shear strength contribution is affected by some disturbance;

** beams which were subjected to pre-cracking 


\section{FIGURE CAPTIONS}

Fig. 1. Possible failure modes of an NSM FRP strip: (a) debonding, (b) laminate tensile rupture, (c) concrete semi-conical fracture, (d) mixed shallow semi-cone plus debonding.

Fig. 2. RC beam web: a) axonometric view of the adopted schematization and b) shear loading process.

Fig. 3. Interaction among adjacent strips: a) axonometric view and b) section parallel to the CDC plane.

Fig. 4. NSM shear strength contribution evaluation: flow chart.

Fig. 5. Single Strip Contribution: (a-b) iterative procedure for searching the equilibrium condition in the surrounding concrete; (c) evaluation of the progressive concrete fracture capacity for a single slip in the simple case of orthogonality between strips and CDC and large spacing and (d) in presence of interaction between adjacent strips not orthogonal to the CDC plane.

Fig. 6. Assumed local bond stress-slip relationship: relevant phases of the failure occurring within the adhesive layer.

Fig. 7. Debonding process for an infinite bond length. Distribution of slip, bond stress, strip axial stress and force transferred to the surrounding concrete along the transfer length for the bond phases: (a) elastic, (b) softening and (c) softening friction.

Fig. 8. Free Slipping phase of the debonding process for an infinite bond length: distribution of slip, bond stress, stress in the strip and progressively transferred force.

Fig. 9. Bond wave progressing from the loaded end to the free extremity: (a) invariant distribution of shear stress $\tau(x)$ and slip $\delta(x)$ and the corresponding distribution of strip axial stress $\sigma_{f}(x)$ and progressive value of the force transferred to the surrounding concrete through bond stresses for an infinite value of the resisting bond length, (b) distribution of slip, tangential stress and progressive force transferred to concrete for a finite value of the resisting bond length and imposed end slip $\delta_{L i}\left(t_{n}\right)$ and for (c) $\delta_{L i}\left(t_{n+1}\right)$.

Fig. 10. CDC plane: (a) geometrical quantities in $O X Y$ and the ellipse local reference system $o_{j} e_{1 j} e_{2 j} ;(\mathrm{b})$ abscissa values necessary to evaluate the $i$-th ellipse's, both, Linear $\mathcal{A}_{f i}^{\text {lin }}$ and Non Linear area $\mathcal{A}_{f i}^{\text {nlin }}$.

Fig. 11. Appraisal of the proposed model for the beams tested by Dias and Barros (2008) and by Dias et al. (2007).

Fig. 12. Cracking scenario regarding beam 2S-7LI45-II: numerical result for $k=1$ (a), $k=2$ (b), $k=3$ (c), and experimental post-test pictures (d-f). 
Fig. 13. Comparison between numerical and experimental results: as function of the spacing between adjacent strips at $60^{\circ}$ for concrete $f_{c m} 31.1 \mathrm{MPa}$ (a) and $f_{c m} 18.6 \mathrm{MPa}$ (b); group effect for the $3^{\text {rd }}$ configuration (concrete $f_{c m} 31.1 \mathrm{MPa}$ and $\beta 60^{\circ}$ ) (c) and ideal shear strength contribution for a system of NSM with spacing $75 \mathrm{~mm}(\mathrm{~d})$.

Fig. 14. Group effect: (a) a real case with a certain value of the spacing between adjacent strips $\left(s_{f, \text { real }}\right)$, (b) a real case with a reduced value of the spacing $\left(0.5 \cdot s_{f, r e a l}\right)$ and (c) ideal situation corresponding to the real case depicted in (a). 